\section{DORF2R.BAS: Analyzing signal-detection theory rating data in the BASIC programming language}

\section{EDWARD F. ALF, JR., and JOHN M. GROSSBERG San Diego State University, San Diego, California}

A standard method of estimating signal-detection theory parameters and confidence intervals for a rating ROC curve of an individual observer has been the application of a maximum likelihood computer algorithm (e.g., Dorfman, 1982; Dorfman \& Alf, 1969). The present program fills the need for a maximum likelihood solution written in a very simple form of Microsoft BASIC.

The accompanying program was written in Microsoft BASIC on the Epson Geneva, a portable 8-bit machine. It was written to be consistent with MBASIC, GWBASIC, BASICA, and most other forms of BASIC. Simple variable names were chosen so the program would be easily transportable from machine to machine.

The program is very similar to the original program by Dorfman and Alf (1969), with the following changes:

1. The normal curve integral and inverse integral routines were obtained by expanding the normal curve equation in a Taylor series, and iterating until the integral converges to the desired decimal accuracy. This is a needless addition for the present program, since the Geneva has only 6 significant figures in single precision. However, an almost identical version has been written for the Cyber, where convergence is carried to 14 significant figures, primarily for research purposes.

2. When the cumulative probabilities are zero or one, the expected cell value is estimated using the Bayesian rule of succession. This method gives the expected cell probability, assuming arbitrarily that all probabilities are equally likely prior to gathering of the data.

3. Rather than use of ordinary least squares to obtain the initial estimates, a weighted least squares procedure is used that weights each data point according to its stability. Furthermore, error is assumed on both axes, rather than on only the $y$-axis. As a result, the initial estimates are very close to the final ones, and frequently the inital chi-square is smaller than the chi-square value after iterations are complete. Thus, the number of iterations is generally reduced, and, more importantly, the procedure rarely fails to converge.

Address correspondence to Edward F. Alf, Jr., or John M. Grossberg, Department of Psychology, San Diego State University, San Diego, CA 92182-0350.
4. The program estimates the positive $z(k)$ values, rather than the negative of these values. This is simply a minor difference in sign, but it also makes a difference in the signs in the variance-covariance matrix.

5 . The additional indices and their variances provided by Dorfman (1982) are given, together with a few others that may be useful for theoretical purposes. Specifically, the indices DELTA $M, D(E), A(Z)$, and $D(A)$ are as defined by Dorfman, and as presented in Appendix D of Swets and Pickett (1982). In addition, the estimates and variances are given for

SD-The standard deviation of the signal-plusnoise distribution, assuming the noise distribution to have a standard deviation of 1.00 ;

DELTA SD-equal to SD-1;

(DELTA M)/(DELTA SD)-The ratio of the difference in means to the difference in variances for the noise and signal-plus-noise distributions. It is sometimes expected that this ratio will be about 4.00 ;

$\mathrm{Z}(\mathrm{A})$-The normal $z$-score below which the area $\mathrm{A}(\mathrm{Z})$ lies.

Appendix A is the program listing. Appendix B is an example using Subject Number 3 from Appendix D of Swets and Pickett $(1982$, p. 218). This reference also provides guidance that should be helpful in using the program, and provides comparisons of the difference in output.

The program is slow, particularly on a slow computer such as the Epson Geneva. However, even on the Geneva, printing begins about $52 \mathrm{sec}$ after the data have been entered. A compiled version of the program is available on disk for the IBM personal computer and compatibles. The compiled version is considerably faster, completing the iterations in just a few seconds.

Availability. A compiled copy of the program for the IBM PC and compatibles can be obtained by writing either author. To cover the costs of disks, duplication, and postage, please include a check made out to SDSU PSYCHOPHYSICS in the amount of $\$ 6$.

\section{REFERENCES}

Dorfman, D. D. (1982). RSCORE-II. In J. A. Swets \& R. M. Pickett, Evaluation of diagnostic systems: Methods from signal detection theory (pp. 212-232). New York: Academic Press.

DorFMAN, D. D., \& ALF, E., JR. (1969). Maximum likelihood estimation of parameters of signal detection theory and determination of confidence intervals-Rating method data. Joumal of Mathematical Psychology, 6, 487-496.

SwETs, J. A., \& PicketT, R. M. (1982). Evaluation of diagnostic systems: Methods from signal detection theory. New York: Academic Press. 


\section{APPENDIX A}

Listing of DORF2R.BAS

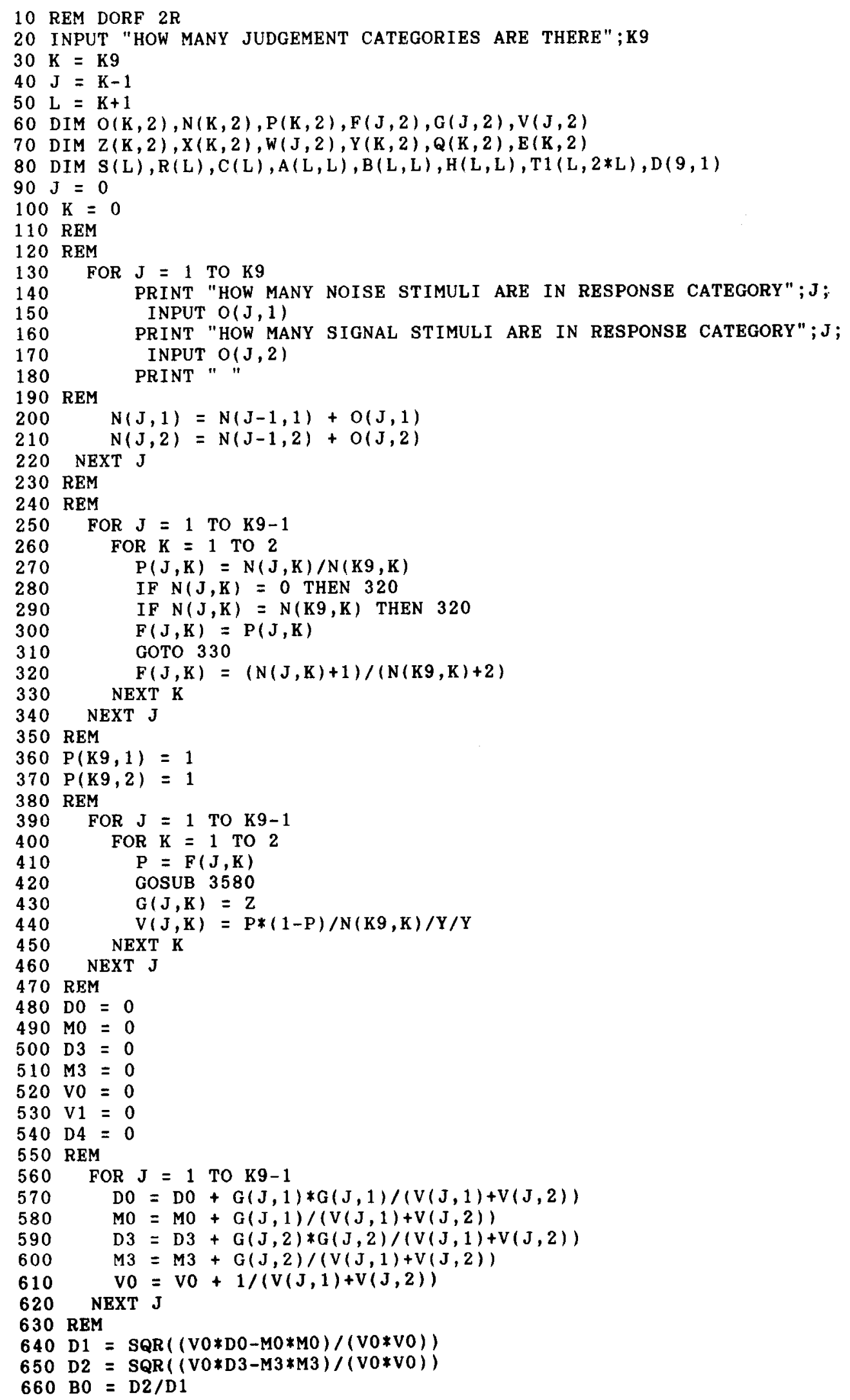




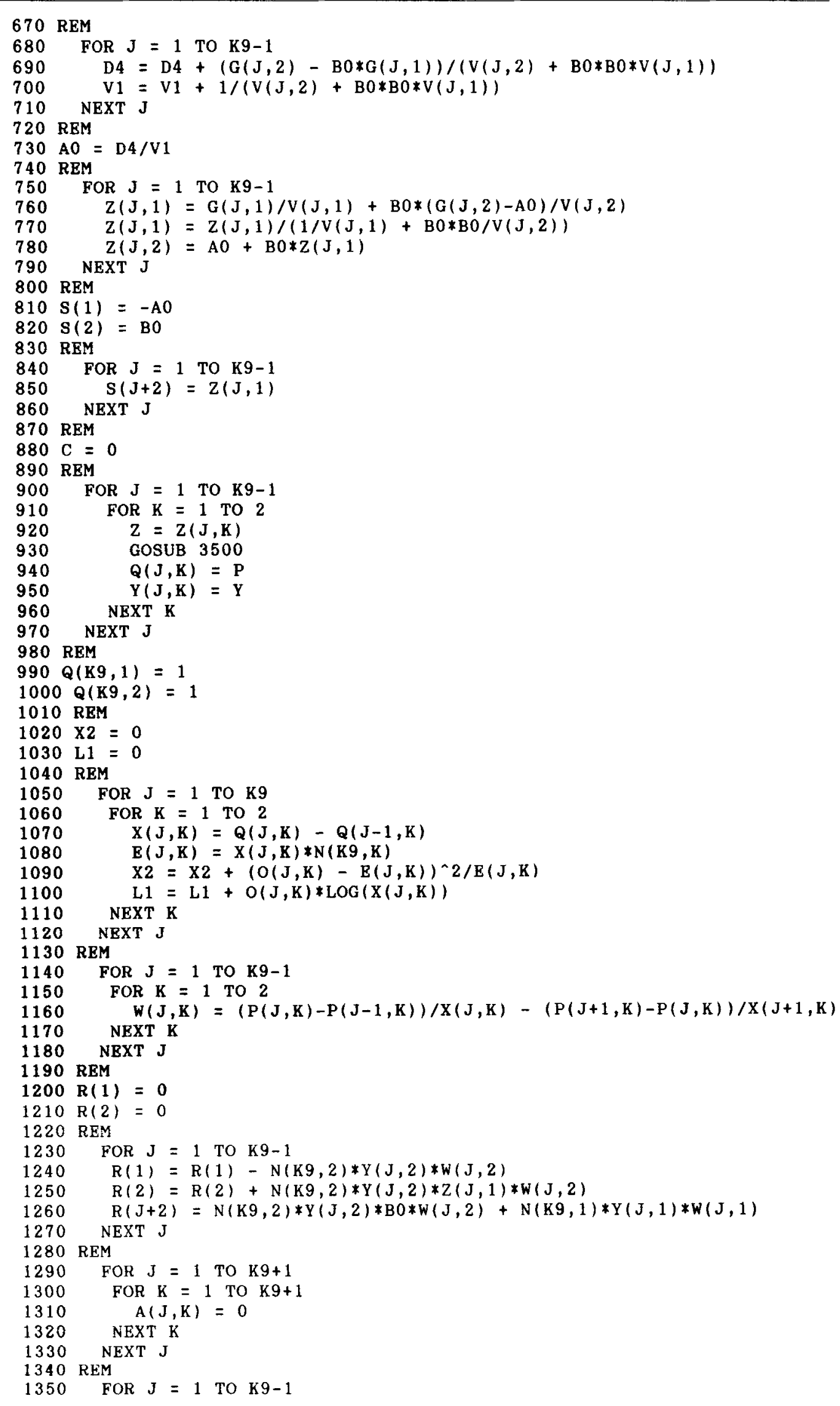




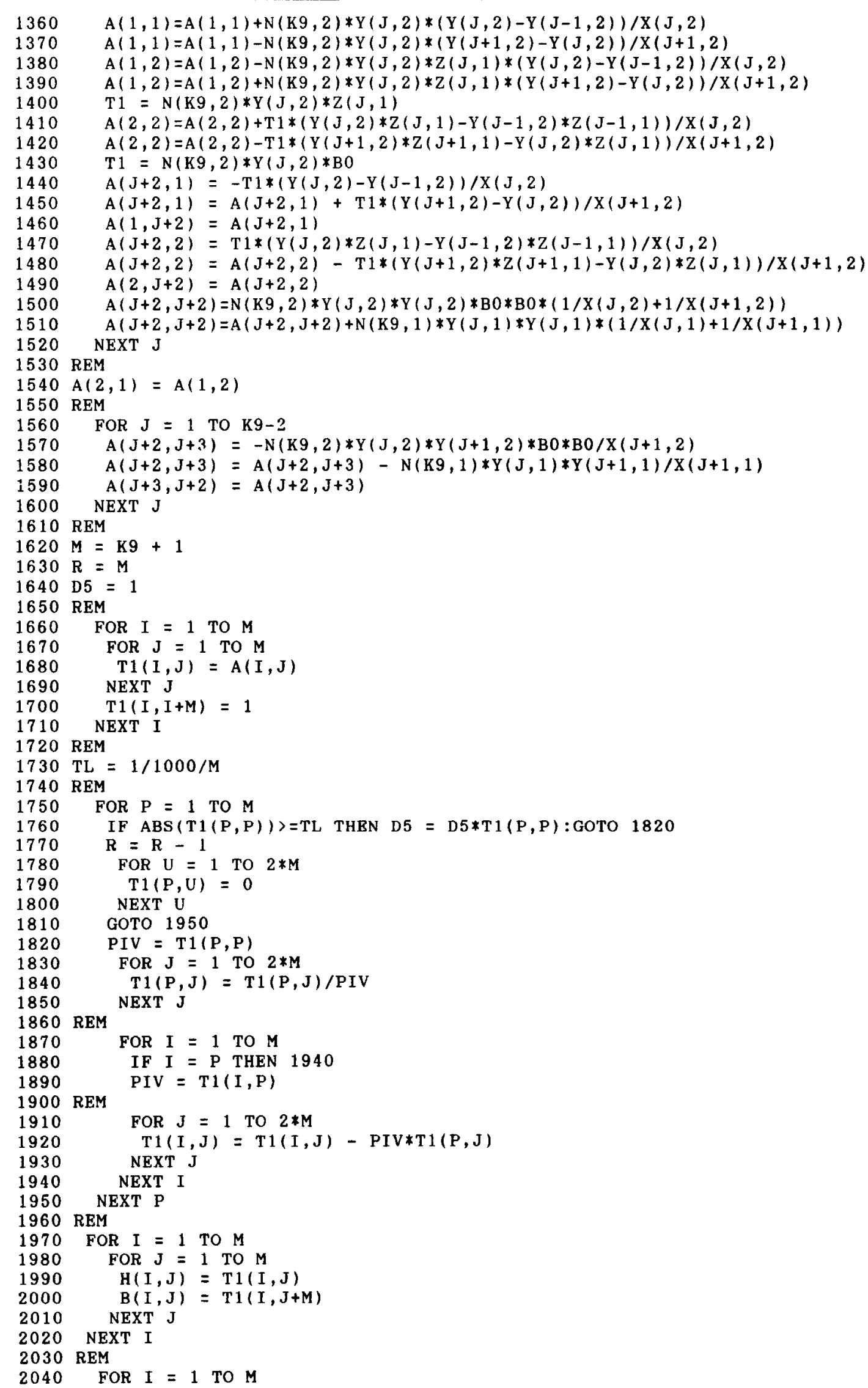




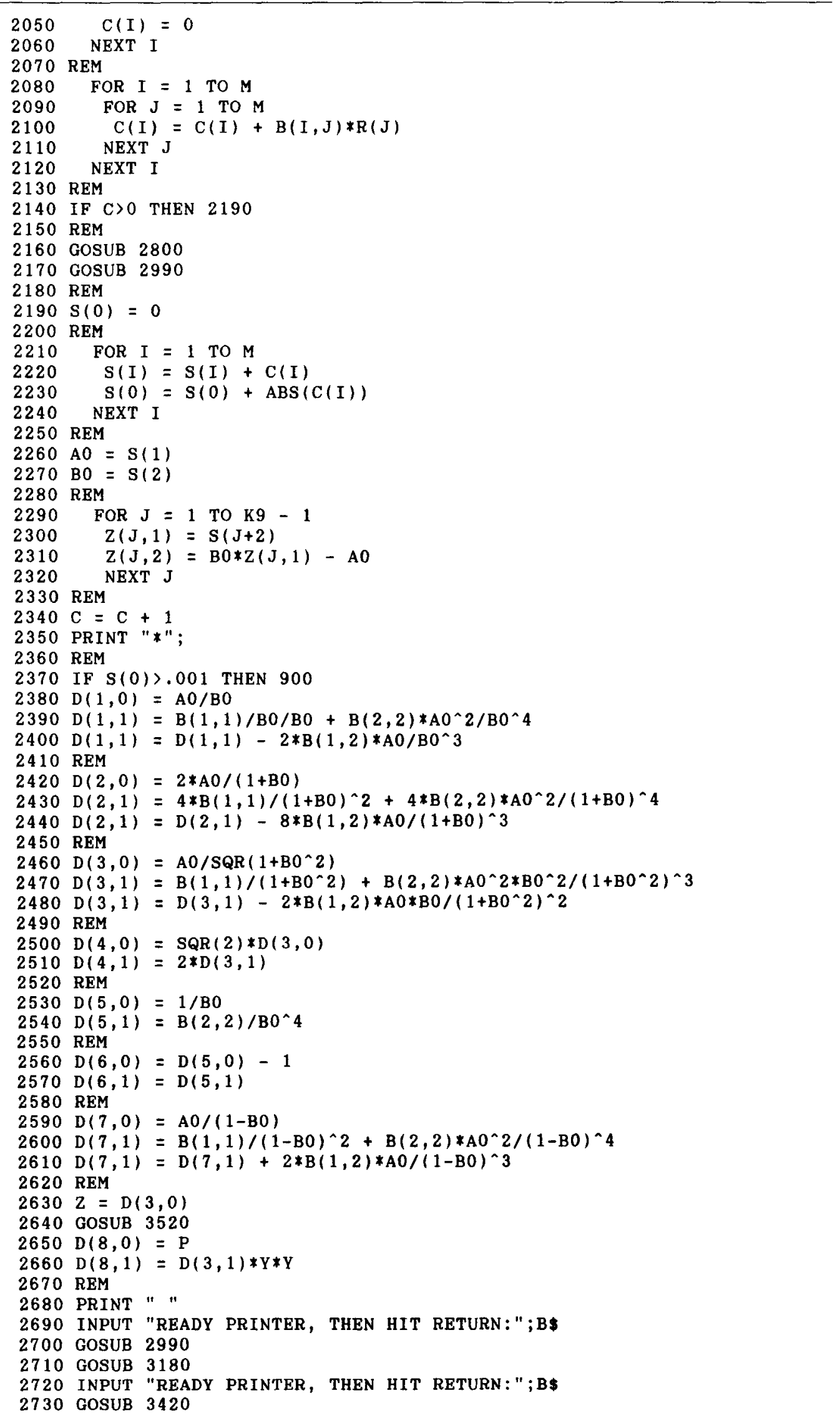


APPENDIX A (Continued)

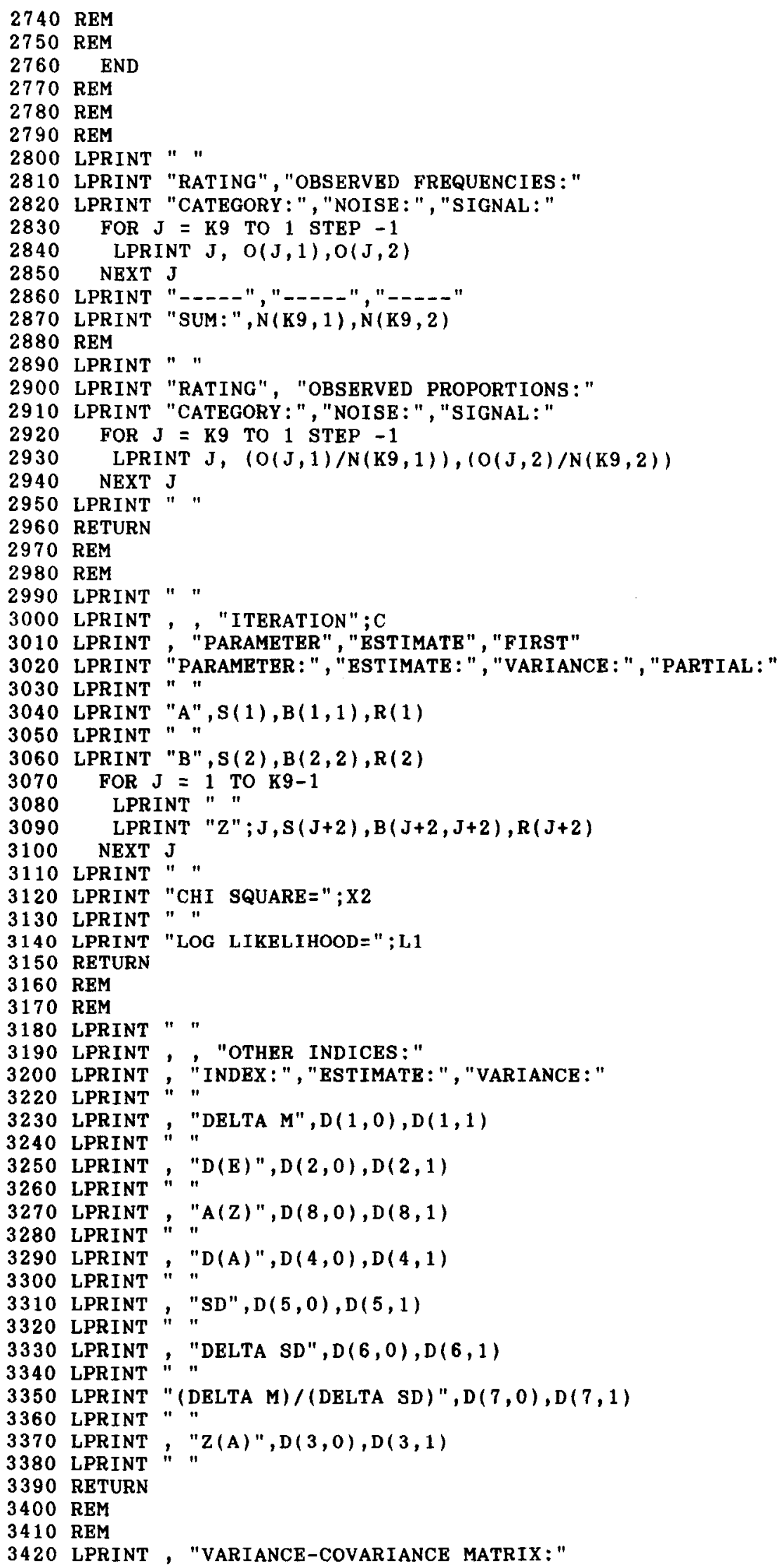




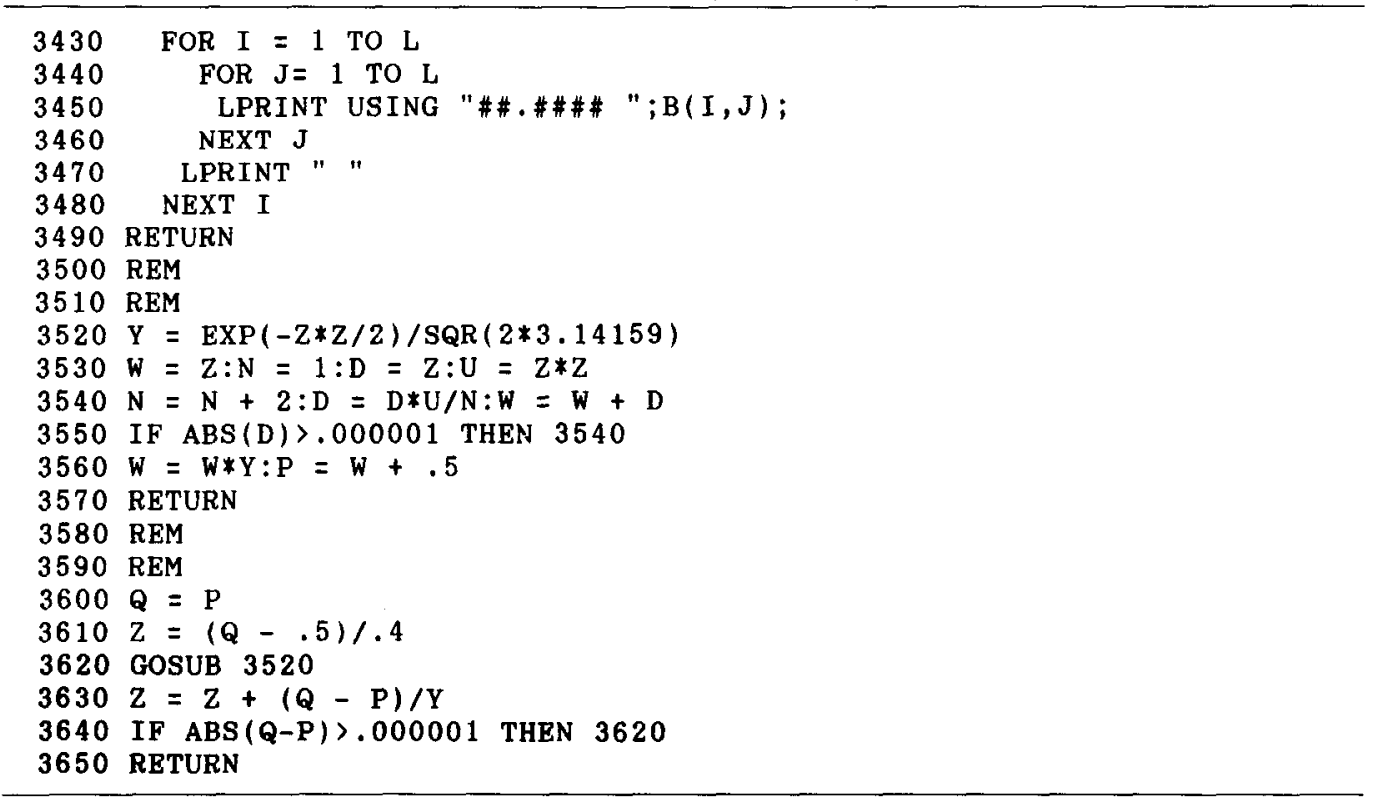

\section{APPENDIX B}

Sample Output for Subject 3 in the User's Manual

\begin{tabular}{|c|c|c|c|}
\hline RATING & \multicolumn{3}{|c|}{ OBSERVED FREQUENCIES: } \\
\hline CATEGORY: & NOISE: & \multicolumn{2}{|l|}{ SIGNAL: } \\
\hline 6 & 47 & \multicolumn{2}{|l|}{348} \\
\hline 5 & 43 & \\
\hline 4 & 42 & \multirow{2}{*}{\multicolumn{2}{|c|}{32}} \\
\hline 3 & 26 & & \\
\hline 2 & 99 & \multirow{2}{*}{\multicolumn{2}{|c|}{44}} \\
\hline 1 & 334 & & \\
\hline---- & ---- & \multirow{2}{*}{\multicolumn{2}{|c|}{597}} \\
\hline SUM : & 591 & & \\
\hline RATING & \multicolumn{3}{|c|}{ OBSERVED PROPORTIONS: } \\
\hline CATEGORY: & NOISE : & \multicolumn{2}{|l|}{ SIGNAL: } \\
\hline 6 & $7.952622 \mathrm{E}-02$ & \multicolumn{2}{|l|}{$\begin{array}{l}\text { SIGNAL: } \\
.5829146\end{array}$} \\
\hline 5 & $7.275803 \mathrm{E}-02$ & \multirow{2}{*}{\multicolumn{2}{|c|}{.1105528}} \\
\hline 4 & $7.106599 \mathrm{E}-02$ & \multirow{2}{*}{$\begin{array}{l}5.360134 \mathrm{E}-02 \\
4.355109 \mathrm{E}-02\end{array}$} & \\
\hline 3 & $4.399323 E-02$ & \multirow{2}{*}{\multicolumn{2}{|c|}{$\begin{array}{l}4.355109 \mathrm{E}-02 \\
7.370184 \mathrm{E}-02\end{array}$}} \\
\hline 2 & .1675127 & & \\
\hline 1 & .5651438 & \multicolumn{2}{|l|}{.1356784} \\
\hline & & \multicolumn{2}{|l|}{ ITERATION 0} \\
\hline & PARAMETER & ESTIMATE & FIRST \\
\hline PARAMETER : & ESTIMATE: & VAR I ANCE : & PART IAL: \\
\hline A & 1.22866 & $6.391967 \mathrm{E}-03$ & -1.241581 \\
\hline B & .716576 & $4.27494 \mathrm{E}-03$ & 1.736123 \\
\hline $\mathrm{Z} 1$ & .1679222 & $2.672896 \mathrm{E}-03$ & .9062052 \\
\hline 22 & .6098079 & $2.748772 E-03$ & -10.40587 \\
\hline 23 & .7698475 & $2.929841 \mathrm{E}-03$ & 14.39708 \\
\hline 24 & 1.019365 & $3.472101 \mathrm{E}-03$ & -8.004211 \\
\hline
\end{tabular}




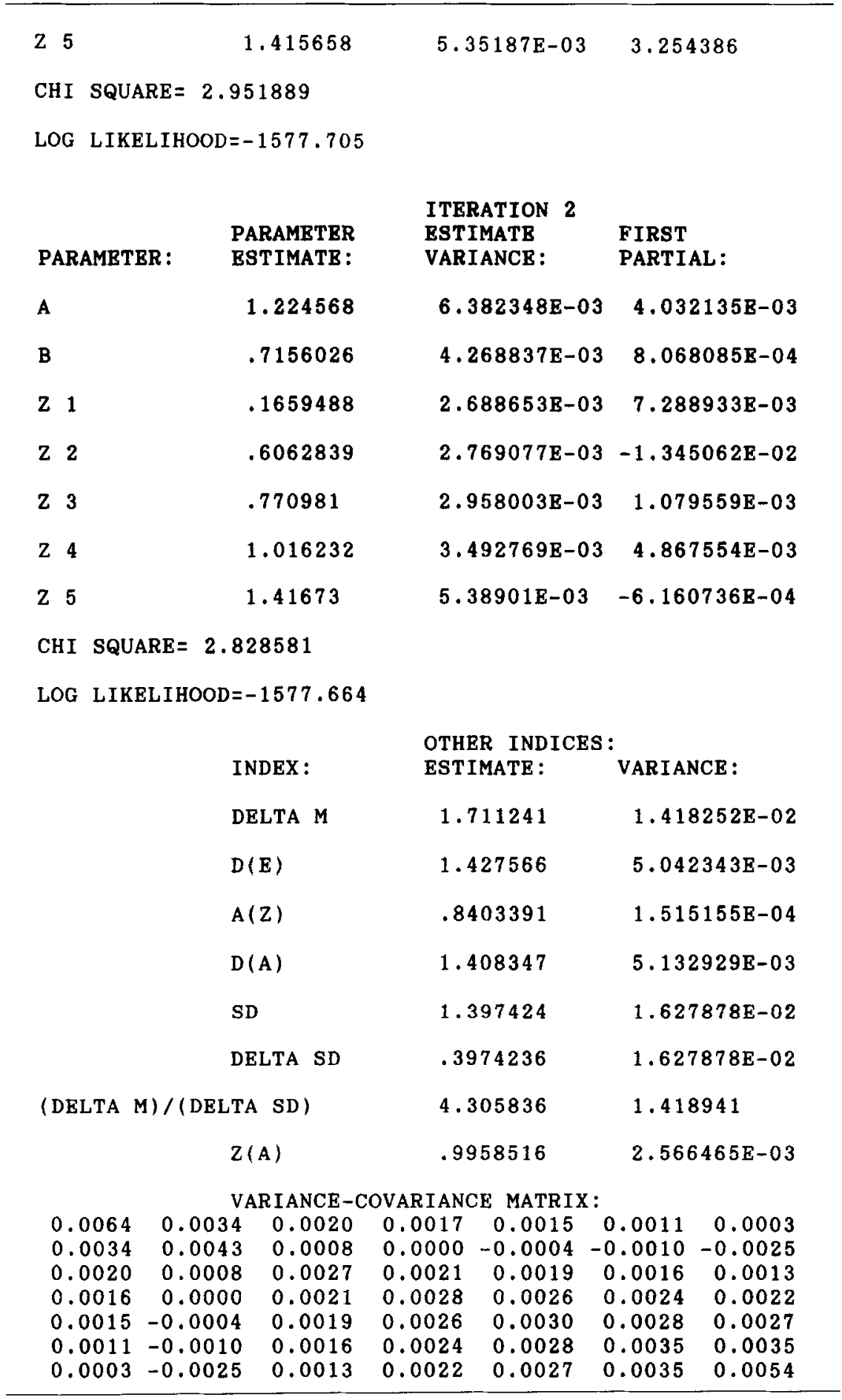

